Received: 14.08 .2019

Revised: 10.02 .2020

Accepted: 21.02 .2020

DOI: $10.17804 / 2410-9908.2020 .1 .043-056$

\title{
ACOUSTIC ANISOTROPY AT DIFFERENT STAGES OF THE DEFORMATION PROCESS IN METALS
}

\author{
D. A. Tretyakov \\ Peter the Great Saint-Petersburg Polytechnic University, \\ 29 Politekhnicheskaya St. Petersburg, 195251, Russian Federation \\ (iD https://orcid.org/0000-0002-2349-9516 @ dmitry.tretyakov93@yandex.ru
}

\author{
Corresponding author. E-mail: tretyakov_da@spbstu.ru \\ Address for correspondence: 29 Polytechnicheskaya, St. Petersburg, 195251, Russian Federation \\ Tel.: +7 (812) 55276 60; fax: +7 (812) 5527660
}

The paper investigates acoustic anisotropy arising under inelastic loading of industrial alloy structures. The results of ultrasonic measurements on specimens of an aluminum-manganese alloy indicate a nonlinear nonmonotonic strain dependence of acoustic anisotropy. It cannot be explained in terms of the theory of acoustoplasticity and the Murnaghan nonlinear elastic model. This theory establishes a linear relationship between acoustic anisotropy and plastic strain. The location of the zones of nonmonotonic changes in the value of acoustic anisotropy is compared with the points on the strain curve. The results suggest that that acoustic anisotropy is an indicator of the beginning of various stages of the deformation process in metals.

Keywords: acoustic anisotropy, plastic deformation, ultrasonic waves, nondestructive testing, acoustoplasticity.

\section{Acknowledgment}

The research was financially supported by the Siemens grant program.

\section{References}

1. Biot M.A. The influence of initial stress on elastic waves. Journal of Applied Physics, 1940, vol. 1 , no. 8, pp. 522-530. DOI: 10.1063/1.1712807.

2. Truesdell C. General and exact theory of waves in finite elastic strain. Archive for Rational Mechanics and analysis, 1961, vol. 8, no. 1, pp. 263-296. DOI: 10.1007/BF00277444.

3. Hughes D.S., Kelly J.L. Second-order elastic deformation of solids. Physical Review, 1953, vol. 92, no. 5, pp. 1145-1159. DOI: 10.1103/PhysRev.92.1145.

4. Benson R.W., Raelson V.J. From ultrasonics to a new stress-analysis technique. Acoustoelasticity. Product Eng., 1959, vol. 30, no. 29, pp. 56-59.

5. Bobrenko V.M., Kutsenko A.N., Sheremetikov A.S. Acoustic tensometry. II: Methods and apparatus/survey. Soviet Journal of Nondestructive Testing, 1981, vol. 16, no. 12, pp. 910-924.

6. Bobrenko V.M., Vangeli M.S., Kutsenko A.N. Acoustic Methods of Control of the Stressed State of Machine Design Materials. Kishinev, Shtiintsa Publ., 1981, 148 p. (In Russian).

7. Bobrenko V.M. Ultrasonic methods and devices for inspecting mechanical stresses. Soviet Journal of Nondestructive Testing, 1984, vol. 19, no. 12, pp. 886-891.

8. Guz' A.N., Makhort F.G., Gushcha O.I., Lebedev V.K. Theory underlying the determination of initial stresses from the results of ultrasonic measurements. International Applied Mechanics, 1971, vol. 7, no. 6, pp. 676-679. DOI: 10.1007/BF00888415. 
9. Guz A.N., Makhort F.G., Gushcha O.I., Lebedev V.K. Osnovy ultrazvukovogo nerazrushayushchego metoda opredeleniya napryazheniy $v$ tverdykh telakh [Basics of Ultrasonic Nondestructive Method for Determining the Stresses in Solids]. Kiev, Naukova Dumka Publ., 1974, 106 p. (In Russian).

10. Guz A.N., Makhort F.G., Gushcha O.I. Vvedenie v akustouprugost [Introduction to Acoustoelasticity]. Kiev, Naukova Dumka Publ., 1977, 162 p. (In Russian).

11. Bystrov V.F., Guzovsky V.V., Zolotov V.F., Nikitina N.E. Influence of technological processing of high-strength steel on the coefficients of elastic-acoustic coupling. Soviet Journal of Nondestructive Testing, 1986, no. 7, pp. 92-93. (In Russian).

12. Guzovsky V.V., Zolotov V.F., Karzov G.P., Konyukhov B.A., Nikitina N.E. Improving the accuracy of the acoustic method for determining residual stresses in welded structures. $\mathrm{Su}$ dostroenie, 1988, no. 5, pp. 41-42. (In Russian).

13. Gushcha O.I., Makhort F.G. Acoustic method of determining biaxial residual stresses. International Applied Mechanics, 1976, vol. 12, no. 10, pp. 1010-1013. DOI: 10.1007/BF00885046.

14. Bobrenko V.M., Averbukh I.I. Ultrasonic method of measuring stresses in parts of threaded joints. Soviet Journal of Nondestructive Testing, 1974, vol. 10, no. 1, pp. 59-66.

15. Nikitina N.E. Akustouprugost. Opyt prakticheskogo primeneniya [Acoustoelasticity. Experience of Practical Usage]. Nizhniy Novgorod, TALAM Publ., 2005, 208 p. (In Russian).

16. Nikitina N.E. The effect of the intrinsic anisotropy of material on the accuracy of stress measurement by the method of acoustoelasticity. Russian Journal of Nondestructive Testing, 1996, vol. 32, no. 8, pp. 650-656.

17. Nikitina, N.E. Studies of the stressed state of welded parts by the acoustoelasticity method. Probl. Mashinostr. Nadezhnosti Mash., 1999, no. 4, pp. 70-73.

18. Nikitina N.E. Determination of plane stress states in structural materials by using elastic body waves. Russian Journal of Nondestructive Testing, 1999, vol. 35, no. 1, pp. 41-46.

19. Kamyshev A.V., Nikitina N.E., Smirnov V.A. Measurement of the residual stresses in the treads of railway wheels by the acoustoelasticity method. Russian Journal of Nondestructive Testing, 2010, vol. 46, no. 3, pp. 189-193. DOI: 10.1134/S106183091003006X.

20. Nikitina N.Y., Kamyshev A.V., Kazachek S.V. The application of the acoustoelasticity method for the determination of stresses in anisotropic pipe steels. Russian Journal of Nondestructive Testing, 2015, vol. 51, no. 3, pp. 171-178. DOI: 10.1134/S1061830915030079.

21. Kamyshev A.V., Pasmanik L.A., Smirnov V.A., Modestov V.S., Pivkov A.V. Use of acoustic anisotropy parameter for the analysis of damage accumulation in the area of SW111 during production and operation of steam generators PGV-1000. In: Transactions of the 9-th International Scientific and Technical Conference: Safety assurance of NPP with VVER. Scientific and technical electronic edition, May 19-22, 2015, OKB “Gidropress” Publ., Podolsk, Russia. (In Russian).

22. Pao Y.H. Theory of Acoustoelasticity and Acoustoplasticity. In: Solid Mechanics Research for Quantitative Non-destructive Evaluation, Springer, Dordrecht, 1987, pp. 257-273. DOI: 10.1007/978-94-009-3523-5_16.

23. Pao Y.H., Wu T.T., Gamer U. Acoustoelastic birefringences in plastically deformed solids: Part I: Theory. Journal of Applied Mechanics, 1991, vol. 58, no. 1, pp. 11-17. DOI: 10.1115/1.2897137.

24. Wu T.T., Hirao M., Pao Y.H. Acoustoelastic birefringences in plastically deformed solids. Part II: Experiment. Journal of Applied Mechanics, 1991, vol. 58, no. 1, pp. 18-23. DOI: 10.1115/1.2897148.

25. Kobayashi M. Theoretical study of acoustoelastic effects caused by plastic anisotropy growth. International Journal of Plasticity, 1987, vol. 3, no. 1, pp. 1-20. DOI: 10.1016/07496419(87)90014-3.

26. Kobayashi M. Ultrasonic nondestructive evaluation of microstructural changes of solid materials under plastic deformation. Part I: Theory. International Journal of Plasticity, 1998, vol. 14, no. 6, pp. 511-522. DOI: 10.1016/S0749-6419(98)00005-9. 
27. Kobayashi M. Ultrasonic nondestructive evaluation of microstructural changes of solid materials under plastic deformation. Part II: Experiment and simulation. International Journal of Plasticity, 1998, vol. 14, no. 6, p. 523-535. DOI: 10.1016/S0749-6419(98)00006-0.

28. Murnaghan F.D. Finite deformations of an elastic solid. American Journal of Mathematics, 1937, vol. 59, no. 2, p. 235-260. DOI: 10.2307/2371405.

29. Hirao M., Pao Y. H. Dependence of acoustoelastic birefringence on plastic strains in a beam. The Journal of the Acoustical Society of America, 1985, vol. 77, no. 5, pp. 1659-1664. DOI: $10.1121 / 1.391964$.

30. Ghosh S., Li M., Gardiner D. A computational and experimental study of cold rolling 31. of aluminum alloys with edge cracking. Journal of Manufacturing Science and Engineering, 2004, vol. 126, no. 1, p. 74-82. DOI: 10.1115/1.1645877.

32. Belyaev A.K., Polyanskiy V.A., Semenov A.S., Tretyakov D.A., Yakovlev Y.A. Investigation of the correlation between acoustic anisotropy, damage and measures of the stress-strain state. Procedia Structural Integrity, 2017, vol. 6, pp. 201-207. DOI: 10.1016/j.prostr.2017.11.031.

33. O'Neill B., Maev R.G. Acousto-elastic measurement of the fatigue damage in Waspaloy. Research in Nondestructive Evaluation, 2006, vol. 17, no. 3, pp. 121-135. DOI: 10.1080/09349840600787931.

34. Riedel H., Andrieux F., Walde T., Karhausen K. F. The formation of edge cracks during rolling of metal sheet. Steel Research International, 2007, vol. 78, no. 10-11, pp. 818-824. DOI: $10.1002 /$ srin.200706291.

35. Ivanova Y., Partalin T., Pashkuleva D. Acoustic investigations of the steel samples deformation during the tensile. Russian Journal of Nondestructive Testing, 2017, vol. 53, no. 1, pp. 39-50. DOI: 10.1134/S1061830917010077.

36. Kurashkin K.V., Gonchar A.V. Variation of acoustic characteristics of an aluminum alloy during plastic deformation at room and subzero temperatures. In: AIP Conference Proceedings, AIP Publishing, 2018, vol. 2053, no. 1, pp. 030030. DOI: 10.1063/1.5084391.

37. Alhimenko A.A., Belyaev A.K., Grishchenko A.I., Semenov A.S., Tretyakov D.A., Polyanskiy V.A., Yakovlev Y.A. Propagation of acoustic waves during the control of hydrogen-induced destruction of metals by the acoustoelastic effect. 2018 Days on Diffraction (DD), IEEE, 2018, pp. 11-16. DOI: 10.1109/DD.2018.8553151.

38. Belyaev A.K., Grishchenko A.I., Polyanskiy V.A., Semenov A.S., Tretyakov D.A., Shtukin L. V., Arseniev D.G., Yakovlev Y.A. Acoustic anisotropy and dissolved hydrogen as an indicator of waves of plastic deformation. 2017 Days on Diffraction (DD), IEEE, 2017, pp. 39-44. DOI: 10.1109/DD.2017.8167992.

39. Semenov A.S., Polyanskii V.A., Shtukin L.V., Tretyakov D.A. Effect of Surface Layer Damage on Acoustic Anisotropy. Journal of Applied Mechanics and Technical Physics, 2018, vol. 59, no. 6, pp. 1136-1144. DOI: 10.1134/S0021894418060202.

40. Alekseeva E.L., Belyaev A.K., Pasmanik L.A., Polyanskiy A.M., Polyanskiy V.A., Tretiakov D.A., Yakovlev Y.A. A study of hydrogen cracking in metals by the acoustoelasticity method. In: AIP Conference Proceedings, AIP Publishing, 2017, vol. 1915, no. 1, pp. 030001. DOI: $10.1063 / 1.5017321$.

41. Belyaev A.K., Mansyrev D.E., Polyanskiy V.A., Polyanskiy A.M., Tretyakov D.A., Yakovlev Yu.A. Boundary Layer of Hydrogen Concentration under Plastic Deformation. Diagnostics, Resource and Mechanics of Materials and Structures, 2017, no. 4, pp. 32-43. DOI: 10.17804/2410-9908.2017.4.032-043. Available at: http://dream-journal.org/issues/20174/2017-4_167.html

42. Polyanskiy V.A., Belyaev A.K., Yakovlev Y.A., Polyanskiy A.M., Tretyakov D.A. Influence of the skin effect of plastic deformation on hydrogen accumulation in metals. In: AIP Conference Proceedings, AIP Publishing, 2018, vol. 2053, no. 1, pp. 020011. DOI: 10.1063/1.5084357.

43. Polyanskiy A.M., Polyanskiy V.A., Frolova K.P., Yakovlev Yu.A. Hydrogen diagnostics of metals and alloys. Diagnostics, Resource and Mechanics of Materials and Structures, 2018, no. 6, 
pp. 37-50. DOI: 10.17804/2410-9908.2018.6.037-050. Available at: http://dream-journal.org/ /DREAM_Issue_6_2018_Polyanskiy_A.M._et_al._037_050.pdf 44. GOST R 52890-2007. Non-destructive testing. Evaluation of stresses in material of pipelines by ultrasound method. General requirements. M., Standartnform Publ., 2009. (In Russian). 
Подана в журнал: 14.08 .2019

УДК 620.179.16

DOI: $10.17804 / 2410-9908.2020 .1 .043-056$

\title{
АКУСТИЧЕСКАЯ АНИЗОТРОПИЯ НА РАЗЛИЧНЫХ СТАДИЯХ ДЕФОРМАЦИОННОГО ПРОЦЕССА В МЕТАЛЛАХ
}

\author{
Д. А. Третьяков \\ Санкт-Петербургский политехнический университет Петра Великого, \\ ул. Политехническая, д. 29, г. Санкт-Петербург, Российская Федераиия \\ (iD https://orcid.org/0000-0002-2349-9516 ख dmitry.tretyakov93@yandex.ru \\ Ответственный автор. Электронная почта: tretyakov_da@spbstu.ru \\ Адрес для переписки: ул. Политехническая, д. 29, 195251, г. Санкт-Петербург, Российская Федерация \\ Тел.: +7 (812) 552-76-60; факс: +7 (812) 552-76-60 \\ Работа посвящена исследованию акустической анизотропии, возникающей при не- \\ упругом нагружении конструкций из промышленного проката. Результаты ультразвуковых \\ измерений, проведенных на образцах из алюминиево-марганцевого сплава, указывают на \\ существование нелинейной немонотонной зависимости акустической анизотропии от де- \\ формаций. Она не может быть объяснена в рамках используемой в теории акустопластично- \\ сти нелинейно-упругой модели Мурнагана и полученного на ее основании соотношения, \\ устанавливающего линейную связь акустической анизотропии с величиной пластических \\ деформаций. По результатам сопоставления участков немонотонности полученной экспери- \\ ментальной зависимости с характерными точками на кривой деформирования, выдвинуто \\ предположение о том, что акустическая анизотропия является индикатором различных ста- \\ дий деформационного процесса в металлах.
}

Ключевые слова: акустическая анизотропия, пластические деформации, ультразвуковые волны, неразрушающий контроль, акустопластичность.

\section{1. Введение}

Существование ненулевой акустической анизотропии - фазового сдвига, возникающего между скоростями поперечных волн взаимно ортогональной поляризации в конструкционных материалах, - является объектом исследований уже более 80 лет.

Одним из первых, кто рассмотрел влияние напряжений на характер распространения акустических волн на теоретическом уровне, был Био [1]. Трусделл получил общее условие распространения волн в напряженной среде в случае нелинейно упругого материала [2]. Это позволило предложить идею использования скоростей поперечных волн для оценки величины механических напряжений. Хьюз и Келли экспериментально обнаружили линейную зависимость скоростей поперечных ультразвуковых волн от величин напряжений в случае одноосного нагружения и гидростатического давления [3]. Это явление в работах Бенсона и Рилсона получило название акустоупругого эффекта [4]. Так были заложены основы для нового направления, связанного с исследованием акустической анизотропии в различных модельных средах и существующих конструкционных материалах.

Акустическая анизотропия рассчитывается по определению согласно следующей формуле:

$$
a=2\left(v_{1}-v_{2}\right) /\left(v_{1}+v_{2}\right)
$$


где $v_{1}$ и $v_{2}$ - скорости поперечных волн взаимно ортогональной поляризации. Разработка промышленного метода измерения механических напряжений при упругих деформациях конструкций с помощью акустической анизотропии велась на территории СССР большим числом научных групп, в том числе коллективами из ВНИИНК (г. Кишинев) [5-7], из Института механики им. С.П. Тимошенко (г. Киев) [8-10] и Нижегородского филиала Института машиноведения им. А.А. Благонравова РАН [11-12]. Были сформулированы рекомендации по выбору направления поляризации поперечных волн в случае одноосного и двухосного напряженно-деформированного состояния [13], проведены измерения напряжений в болтах и резьбовых соединениях [14]. В постсоветское время был сформулирован и стандартизирован метод акустоупругости [15], большой вклад в дело практического применения которого внесла Н.Е. Никитина [16-18] и коллектив сотрудников Нижегородского филиала ИМАШ РАН. Были предложены методики измерения напряжений в вагонных колесах и рельсах [19], трубопроводах [20], парогенераторах [21] и других элементах энергетического оборудования.

Наряду с изучением акустоупругого эффекта нашел широкое распространение подход, связанный с использованием акустической анизотропии для оценки пластических деформаций [22-27]. Теоретические соотношения, связывающие акустическую анизотропию с главными пластическими деформациями, были получены на основе нелинейно-упругой модели Мурнагана [28]. В программной работе [29] был установлен линейный характер связи между акустической анизотропией и пластическими деформациями :

$$
a=a_{0}+a_{1}\left(\varepsilon_{1}^{P}-\varepsilon_{2}^{P}\right)+C_{A}\left(\sigma_{1}-\sigma_{2}\right),
$$

где $\varepsilon_{1}^{P}, \varepsilon_{2}^{P}$ - главные пластические деформации; $\sigma_{1}$ и $\sigma_{2}$ - главные напряжения; $a_{1}, C_{A}-$ константы материала, не зависящие от пластических деформаций [29]; $a_{0}$ - собственная акустическая анизотропия материала, обусловленная влиянием структуры, начального текстурирования и иных факторов.

Появившиеся в последнее время экспериментальные [30-35] и теоретические [36, 37] результаты ставят под сомнение универсальность данного подхода для отличных от рассматриваемого авторами [29] случая малых пластических деформаций. В частности, отмечается существование поверхностного эффекта акустической анизотропии в промышленном прокате [38], влияние коррозионного [39] и водородного [40-42] растрескивания металлов на величину акустической анизотропии.

Необходимо исследовать характер влияния неупругих факторов на акустическую анизотропию в широком диапазоне упругопластических деформаций вплоть до шейкообразования и разрушения на примере реальных технических конструкций, изготовленных из промышленного проката.

\section{2. Материалы и методика исследования}

Исследования акустической анизотропии осуществлялись на корсетных образцах из алюминиево-марганцевого сплава АМц. Они были вырезаны поперек направления проката и имели геометрические размеры (рис. 1).

Измерения акустической анизотропии осуществлялись с помощью ультразвукового прибора ИН-5101А (рис. 2), предназначенного для расчета механических напряжений согласно методике, описанной в стандарте [43]. Прибор позволяет осуществлять излучение и прием объемных ультразвуковых волн частотой 5 МГц с помощью контактного датчика (рис. 3). В состав датчика входят три пьезопреобразователя, отвечающих за возбуждение поперечных волн взаимно ортогональной поляризации и продольных волн. 


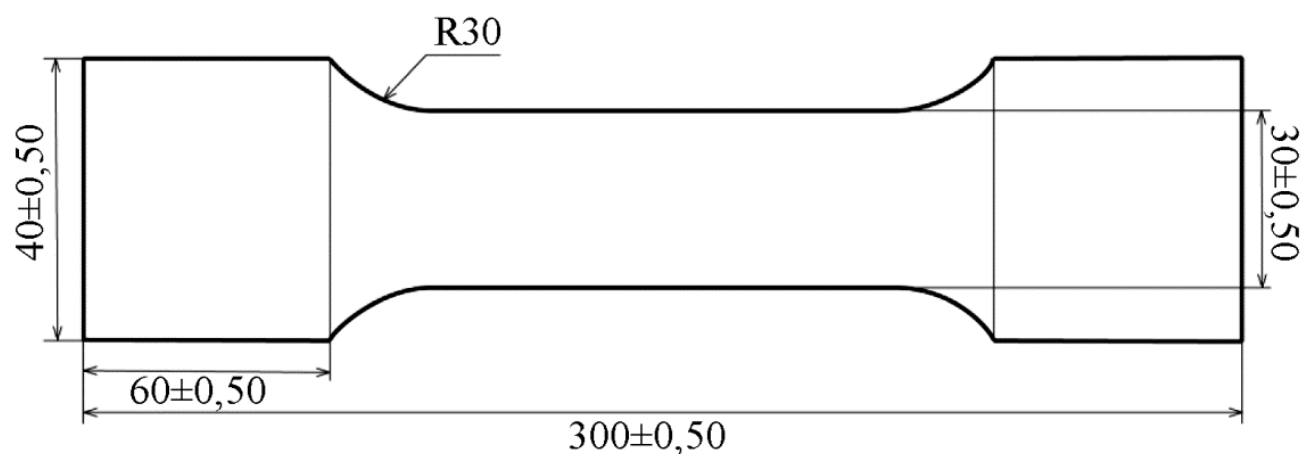

Рис. 1. Геометрические размеры корсетных алюминиевых образцов марки АМц

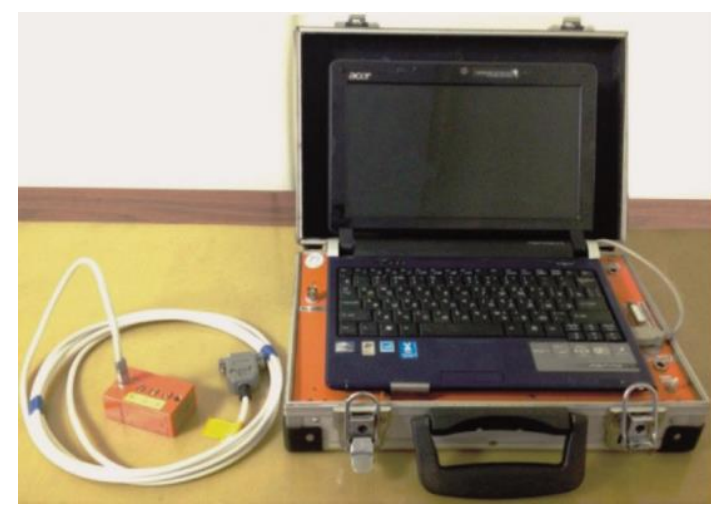

Рис. 2. Прибор для измерения акустической анизотропии ИН-5101А

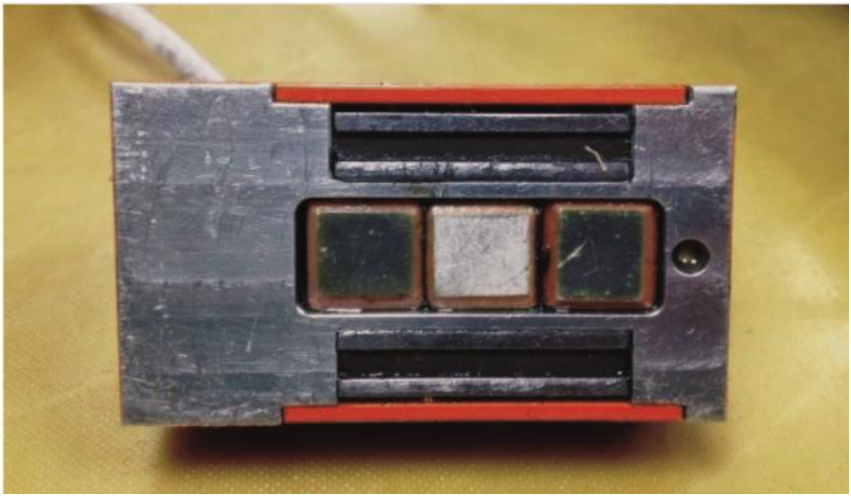

Рис. 3. Акустический датчик, обеспечивающий излучение и прием ультразвуковых волн с частотой 5 МГц

Расчет акустической анизотропии $a$, \% осуществлялся согласно формуле (3) путем измерения с точностью $10^{-9}$ секунды временных задержек $t_{1}$ и $t_{2}$ между пакетами зондирующих импульсов поперечных волн, регистрируемых после многократного отражения от поверхности образца, противоположной той, на которую устанавливается датчик:

$$
a=2\left(t_{1}-t_{2}\right) /\left(t_{1}+t_{2}\right)
$$

\section{3. Результаты и их обсуждение}

Проведенные исследования были разделены на два этапа. На первом этапе был изучен характер зависимости акустической анизотропии от упругопластических деформаций вплоть до момента образования шейки. Измерения $a$, \% осуществлялись в центральной точке рабочей части образцов при жестком одноосном ступенчатом нагружении. Механические испытания проводились на гидравлической машине INSTRON-8801. На этом этапе ультразвуковой датчик был ориентирован вдоль оси каждого из образцов таким образцом, чтобы скорость поперечной волны $v_{l}$ была поляризована вдоль направления действия растягивающей нагрузки.

На рис. 5 представлена полученная по результатам ультразвуковых измерений зависимость акустической анизотропии (ее значения для наглядности были взяты с обратным знаком) от логарифмических деформаций $\varepsilon$, \%. Красными маркерами на рис. 5 отмечены значения акустической анизотропии, соответствующие полным осевым деформациям $\varepsilon$, \% образца, при которых на диаграмме деформирования достигались предел текучести и предел временного сопротивления, осредненные для всей партии образцов (рис. 4). 


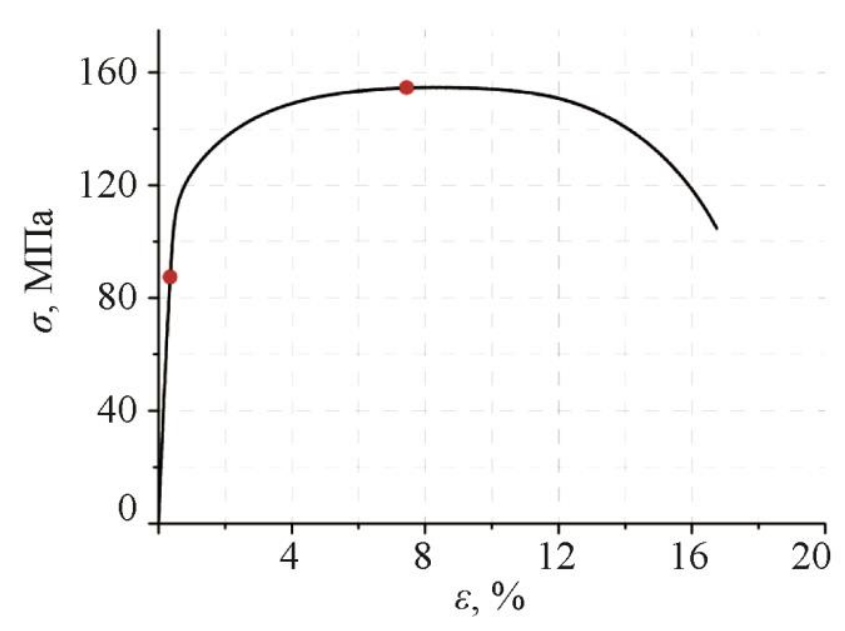

Рис. 4. Диаграмма деформирования для случая одноосного растяжения образцов

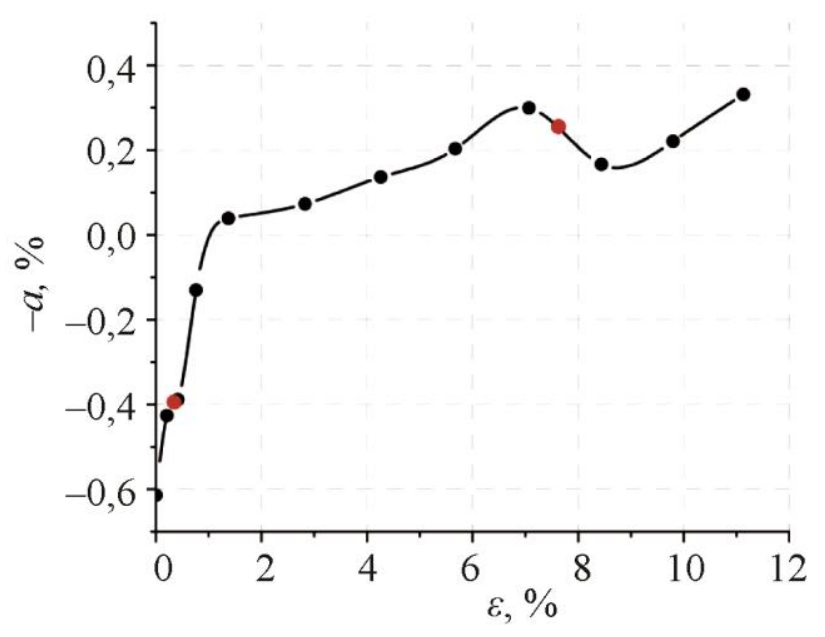

Рис. 5. Зависимость акустической анизотропии $-a, \%$ от деформаций $\varepsilon, \%$

Расположение участков немонотонности графика $-a, \%$ (рис. 5) коррелирует с расположением характерных точек на диаграмме деформирования на рис. 4. Можно предположить, что природой немонотонного характера изменения акустической анизотропии является наступление различных стадий деформационного процесса - начала пластического течения и образования шейки в процессе одноосного растяжения. Следует отметить, что первый участок немонотонности (на рис. 5), приходящийся на случай малых неупругих деформаций, не подчиняется линейной зависимости акустической анизотропии от пластических деформаций, устанавливаемой формулой (2).

На втором этапе исследовались совместное влияние текстуры, вызванной прокатом, и упругопластических деформаций на результаты измерения акустической анизотропии $a, \%$ в случае различной ориентации акустического датчика относительно направления действия растягивающей нагрузки. Случай имеет прикладной интерес, поскольку зачастую определение направления действия главных напряжений в технических конструкциях со сложной геометрией оказывается затруднительным.

В случае исследуемых образцов, вырезанных вдоль направления проката, можно сделать предположение о том, что при одноосном растяжении ориентация осей анизотропии механических свойств совпадает с ориентацией осей главных напряжений.

Измерения акустической анизотропии $a$, \% на втором этапе осуществлялись в центральной точке образцов при отклонениях ультразвукового датчика (рис. 3) на углы, отсчитываемые от $0^{\circ}$ (вдоль оси образцов) против часовой стрелки с шагом $22,5^{\circ}$ вплоть до $157,5^{\circ}$. Зависимости, представленные на рис. 6 и 7, получены для логарифмических значений локальных деформаций $\varepsilon$, \%, измеренных в области исследования акустической анизотропии с помощью высокоточного тензодатчика с базой измерения 10 мм и точностью $10^{-4} \mathrm{Mм}$.

На рис. 6 представлено семейство угловых диаграмм акустической анизотропии, построенных по результатам акустических измерений на различных этапах одноосного растяжения одного из исследуемых образцов. 


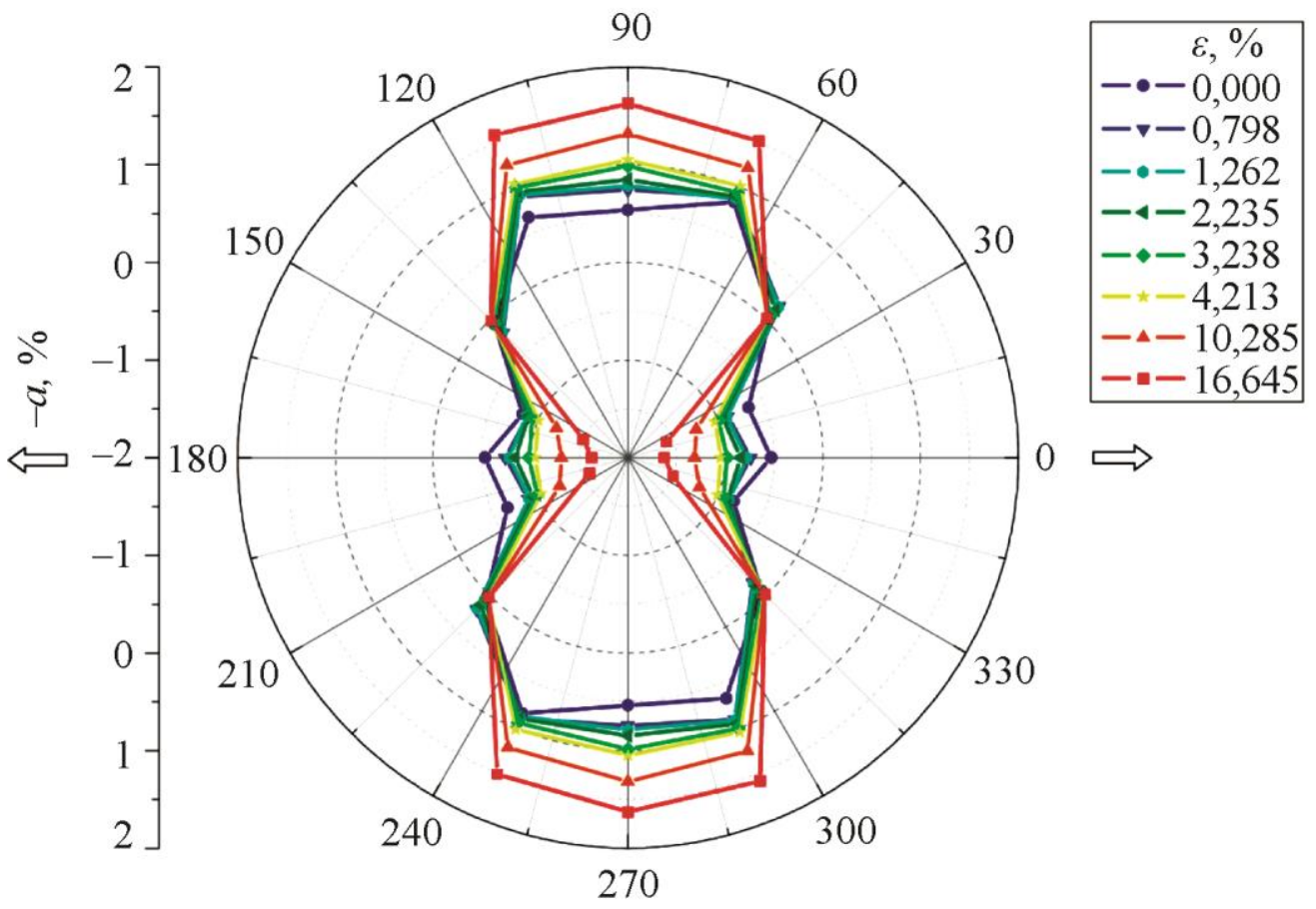

Рис. 6. Угловые диаграммы акустической анизотропии $a$, \%, полученные при одноосном ступенчатом растяжении алюминиевого образца для восьми направлений поляризации поперечных волн, полученных путем поворота ультразвукового датчика относительно оси образца

Неравномерный характер распределения величины акустической анизотропии $a$, \% в различных направлениях, образующий характерную форму угловых диаграмм в виде «восьмерок» (рис. 6) и наблюдаемый в том числе и до механического нагружения образца, обусловлен начальным текстурированием материала, вызванным прокатом. В случае проведенных испытаний он сохраняется на всех этапах нагружения образца, влияние начального текстурирования на величину акустической анизотропии $a$, \% сопоставимо с суммарным вкладом упругих и пластических деформаций (рис. 6).

Во-первых, это означает, что для проведения корректной оценки механических напряжений, даже в случае наличия только упругих деформаций и в соответствии со стандартом [43], вклад величины начальной анизотропии $a_{0}$ в случае диагностики конструкций из промышленного проката не может быть учтен в виде константы в формуле (2). Требуется исследование величины акустической анизотропии $a_{0}$ как функции от угла поворота системы из ортогонально поляризованных поперечных волн.

Во-вторых, построение угловых диаграмм акустической анизотропии (рис. 6) может быть использовано для определения в случае проката заранее неизвестной ориентации осей анизотропии механических свойств материала путем неразрушающего контроля.

Зависимости на рис. 7 получены путем более подробного рассмотрения углов $45^{\circ}$, $67,7^{\circ}, 90^{\circ}$ и $112,5^{\circ}$ (рис. 6), для которых наблюдался положительный знак акустической анизотропии. Значения акустической анизотропии для углов $135^{\circ}, 157,5^{\circ}, 0^{\circ}$ и $22,5^{\circ}$ соответственно равны выбранным на рис. 7 по модулю и противоположны им по знаку. 


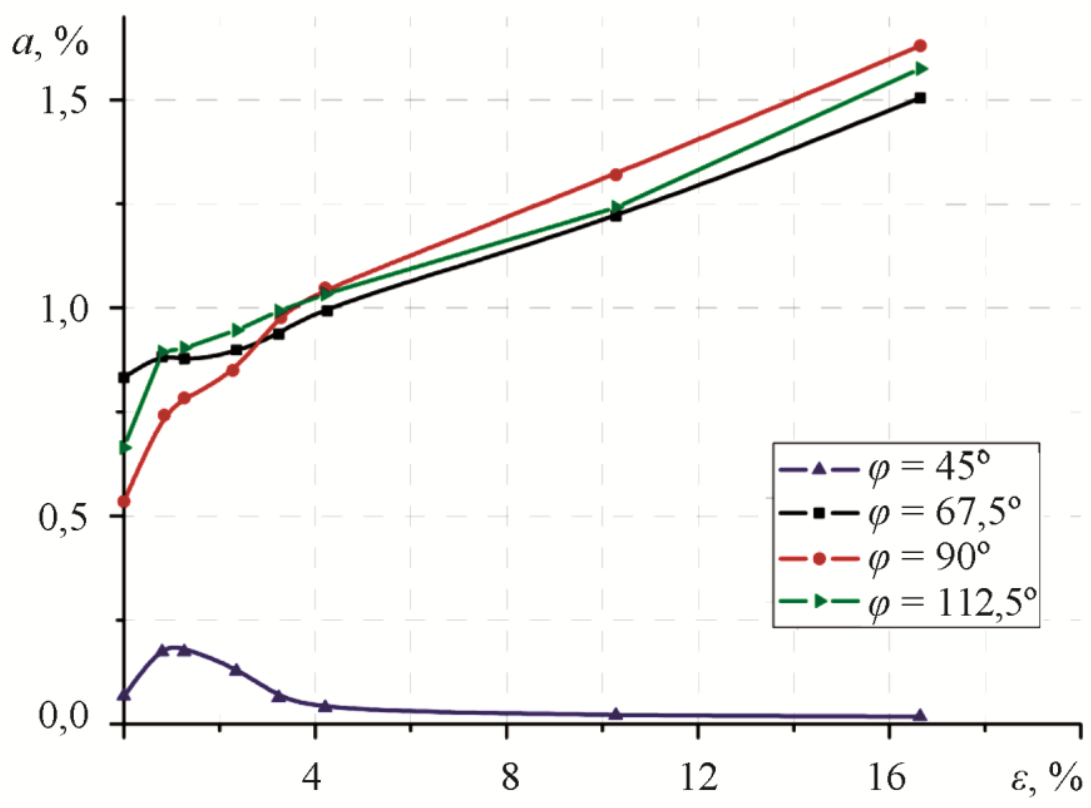

Рис. 7. Зависимости акустической анизотропии $a, \%$ от локальных деформаций $\varepsilon, \%$ для углов поворота ультразвукового датчика, при которых наблюдается положительный знак акустической анизотропии при испытаниях на одноосное растяжение алюминиевого образца

Зависимости, представленные на рис. 7, свидетельствуют о существовании таких направлений поворота датчика, при которых величина акустической анизотропии становится практически нечувствительной к величине упругопластических деформаций выше определенного уровня. В частности, для угла в $45^{\circ}$ и получаемых путем поворота относительно него на $90^{\circ}$ максимальное изменение величины акустической анизотропии не превышает $0,125 \%$ и с ростом деформаций выше 0,8 \% по абсолютной величине начинает монотонно стремиться к нулю. В то время как для углов $67,7^{\circ}, 90^{\circ}$ и $112,5^{\circ}$ максимальные изменения величины акустической анизотропии, наблюдаемые на последнем этапе измерений при величине локальных деформаций 16,6 \%, равны 0,671 \%, 1,095 \% и 0,911\% соответственно.

В случае, представленном на рис. 7, максимальные и минимальные значения акустической анизотропии, полученные в результате испытаний на последнем этапе нагружения образца для углов $90^{\circ}$ и $45^{\circ}$, отличаются между собой по величине в 89,78 раз. Данный экспериментальный факт может приводить к значительным ошибкам в расчете напряжений и оценке величины деформаций конструкции при измерениях согласно стандарту [43]. Контроль анизотропного характера изменения акустической анизотропии в различных направлениях поляризации поперечных ультразвуковых волн может быть произведен путем построения угловых диаграмм акустической анизотропии.

\section{4. Заключение}

Результаты проведенных в работе экспериментальных исследований указывают на существование нелинейной зависимости акустической анизотропии от пластических деформаций. Наблюдается локальный немонотонный характер изменения акустической анизотропии при величинах деформаций, соответствующих значениям пределов текучести и временного сопротивления, осредненным для образцов, изготовленных из одной партии и прошедших испытания при одинаковых условиях нагружения. Можно предположить, что природой немонотонного характера изменения акустической анизотропии является наступление различных стадий деформационного процесса, и акустическая анизотропия может быть использована в качестве их индикатора. 
Наблюдается существенное влияние начального текстурирования на величину и характер распределения акустической анизотропии в различных направлениях поляризации поперечных ультразвуковых волн относительно направления проката и действия главных растягивающих напряжений. Угловые диаграммы, построенные для углов, отсчитываемых против часовой стрелки с шагом $22,5^{\circ}$ от направления вдоль оси образца, имеют характерный вид «восьмерок», наблюдаемый до механических испытаний и на всех этапах нагружения вплоть до его разрушения. Вклад начальной акустической анизотропии исследуемых образцов оказывается сопоставимым с суммарным вкладом упругих и пластических деформаций в величину акустической анизотропии. Этот факт требует рассмотрения начальной акустической анизотропии $a_{0}$ не как константы, а как функции от угла поворота системы из ортогонально поляризованных поперечных волн. Определение заранее неизвестной ориентации осей анизотропии механических свойств материала в случае промышленного проката может быть произведено путем построения угловых диаграмм акустической анизотропии.

Установлен факт существования углов поворота акустического датчика относительно направления действия растягивающей нагрузки, при которых величина акустической анизотропии становится практически нечувствительной к величине неупругих деформаций выше определенного уровня. Наблюдается разница в 89,78 раз между максимальными и минимальными значениями акустической анизотропии, измеренными для углов поворота датчика $90^{\circ}$ и $45^{\circ}$ при одинаковой величине упругопластических деформаций. Значительное расхождение в значениях акустической анизотропии, наблюдаемое на всех этапах нагружения образца, может приводить к ошибкам в расчете механических напряжений с помощью акустической анизотропии. Контроль величины акустической анизотропии в различных направлениях поляризации поперечных ультразвуковых волн также может осуществляться путем построения угловых диаграмм акустической анизотропии.

Полученные результаты позволяют сформулировать новые диагностические признаки для проведения оценки пластических деформаций по результатам ультразвуковых измерений акустической анизотропии. Они могут быть также использованы для усовершенствования существующей методики оценки напряженно-деформируемого состояния промышленных конструкций и деталей машин с помощью метода акустоупругости в части определения вклада начального текстурирования в интегральную величину акустической анизотропии.

\section{Благодарность}

Исследование выполнено при финансовой поддержке стипендиальной программь компании Siemens.

\section{Литература}

1. Biot M. A. The influence of initial stress on elastic waves // Journal of Applied Physics. 1940. - Vol. 1, no. 8. - P. 522-530. - DOI: 10.1063/1.1712807.

2. Truesdell C. General and exact theory of waves in finite elastic strain // Archive for Rational Mechanics and analysis. - 1961. - Vol. 8, no. 1. - P. 263-296. - DOI: 10.1007/BF00277444.

3. Hughes D. S., Kelly J. L. Second-order elastic deformation of solids // Physical Review. 1953. - Vol. 92, no. 5. - P. 1145-1159. - DOI: 10.1103/PhysRev.92.1145.

4. Benson R. W., Raelson V. J. From ultrasonics to a new stress-analysis technique // Acoustoelasticity. Product Eng. - 1959. - Vol. 30, no. 29. - P. 56-59.

5. Bobrenko V. M., Kutsenko A. N., Sheremetikov A. S. Acoustic tensometry. II - Methods and apparatus/survey // Soviet Journal of Nondestructive Testing. - 1981. - Vol. 16, no. 12. P. 910-924.

6. Бобренко В. М., Вангели М. С., Куценко А. Н. Акустические методы контроля напряженного состояния материала деталей машин. - Кишинев : Штиинца, 1981. - 148 с. 
7. Bobrenko V. M. Ultrasonic methods and devices for inspe6cting mechanical stresses // Soviet Journal of Nondestructive Testing. - 1984. - Vol. 19, no. 12. - P. 886-891.

8. Theory underlying the determination of initial stresses from the results of ultrasonic measurements / A. N. Guz', F. G. Makhort, O. I. Gushcha, V. K. Lebedev // International Applied Mechanics. - 1971. - Vol. 7, no. 6. - P. 676-679. - DOI: 10.1007/BF00888415.

9. Основы ультразвукового неразрушающего метода определения напряжений в твердых телах / А. Н. Гузь, Ф. Г. Махорт, О. И. Гуща, В. К. Лебедев. - Киев : Наукова Думка, 1974. $106 \mathrm{c}$.

10. Гузь А. Н., Махорт Ф. Г., Гуща О. И. Введение в акустоупругость. - Киев : Наукова Думка, 1977. - 162 с.

11. Влияние технологической обработки высокопрочной стали на коэффициенты упругоакустической связи / В. Ф. Быстров, В. В. Гузовский, В. Ф. Золотов, Н. Е. Никитина // Дефектоскопия. - 1986. - № 7. - С. 92-93.

12. Повышение точности акустического метода определения остаточных напряжений в сварных конструкциях / В. В. Гузовский, В. Ф. Золотов, Г. П. Карзов, Б. А. Конюхов, Н. Е. Никитина // Судостроение. - 1988. - № 5. - С. 41-42.

13. Gushcha O. I., Makhort F. G. Acoustic method of determining biaxial residual stresses // International Applied Mechanics. - 1976. - Vol. 12, no. 10. - P. 1010-1013. DOI: $10.1007 / \mathrm{BF} 00885046$.

14. Bobrenko V. M., Averbukh I. I. Ultrasonic method of measuring stresses in parts of threaded joints // Soviet Journal of Nondestructive Testing. - 1974. - Vol. 10, no. 1. - P. 59-66.

15. Никитина Н. Е. Акустоупругость. Опыт практического применения. - Н. Новгород : ТАЛАМ, 2005. $-208 \mathrm{c}$.

16. Nikitina N. E. The effect of the intrinsic anisotropy of material on the accuracy of stress measurement by the method of acoustoelasticity // Russian Journal of Nondestructive Testing. 1996. - Vol. 32, no. 8. - P. 650-656.

17. Nikitina, N.E. Studies of the stressed state of welded parts by the acoustoelasticity method // Probl. Mashinostr. Nadezhnosti Mash. - 1999. - No. 4. - P. 70-73.

18. Nikitina N. E. Determination of plane stress states in structural materials by using elastic body waves // Russian Journal of Nondestructive Testing. - 1999. - Vol. 35, no. 1. - P. 41-46.

19. Kamyshev A. V., Nikitina N. E., Smirnov V. A. Measurement of the residual stresses in the treads of railway wheels by the acoustoelasticity method // Russian Journal of Nondestructive Testing. - 2010. - Vol. 46, no. 3. - P. 189-193. - DOI: 10.1134/S106183091003006X.

20. Nikitina N. Y., Kamyshev A. V., Kazachek S. V. The application of the acoustoelasticity method for the determination of stresses in anisotropic pipe steels // Russian Journal of Nondestructive Testing. - 2015. - Vol. 51, no. 3. - P. 171-178. - DOI: 10.1134/S1061830915030079.

21. Use of acoustic anisotropy parameter for the analysis of damage accumulation in the area of SW111 during production and operation of steam generators PGV-1000 / A. V. Kamyshev, L. A. Pasmanik, V. A. Smirnov, V. S. Modestov, A. V. Pivkov // Transactions of the 9-th International scientific and technical conference Safety assurance of NPP with WWER : Scientific and Technical Electronic Edition. - 2015.

22. Pao Y. H. Theory of acoustoelasticity and acoustoplasticity // Solid mechanics research for quantitative non-destructive evaluation. - Springer, Dordrecht, 1987. - P. 257-273. DOI: 10.1007/978-94-009-3523-5_16.

23. Pao Y. H., Wu T. T., Gamer U. Acoustoelastic birefringences in plastically deformed solids. Part I: Theory // Journal of Applied Mechanics. - 1991. - Vol. 58, no. 1. - P. 11-17. DOI: $10.1115 / 1.2897137$.

24. Wu T. T., Hirao M., Pao Y. H. Acoustoelastic birefringences in plastically deformed solids. Part II: Experiment //Journal of Applied Mechanics. - 1991. - Vol. 58, no. 1. - P. 18-23. DOI: $10.1115 / 1.2897148$. 
25. Kobayashi M. Theoretical study of acoustoelastic effects caused by plastic anisotropy growth // International Journal of Plasticity. - 1987. - Vol. 3, no. 1. - P. 1-20. DOI: 10.1016/0749-6419(87)90014-3.

26. Kobayashi M. Ultrasonic nondestructive evaluation of microstructural changes of solid materials under plastic deformation. Part I: Theory // International Journal of Plasticity. - 1998. Vol. 14, no. 6. - P. 511-522. - DOI: 10.1016/S0749-6419(98)00005-9.

27. Kobayashi M. Ultrasonic nondestructive evaluation of microstructural changes of solid materials under plastic deformation. Part II: Experiment and simulation // International Journal of Plasticity. - 1998. - Vol. 14, no. 6. - P. 523-535. - DOI: 10.1016/S0749-6419(98)00006-0.

28. Murnaghan F. D. Finite deformations of an elastic solid // American Journal of Mathematics. - 1937. - Vol. 59, no. 2. - P. 235-260. - DOI: 10.2307/2371405.

29. Hirao M., Pao Y. H. Dependence of acoustoelastic birefringence on plastic strains in a beam // The Journal of the Acoustical Society of America. - 1985. - Vol. 77, no. 5. - P. 1659-1664. DOI: $10.1121 / 1.391964$.

30. Ghosh S., Li M., Gardiner D. A computational and experimental study of cold rolling of aluminum alloys with edge cracking // Journal of Manufacturing Science and Engineering. 2004. - Vol. 126, no. 1. - P. 74-82. - DOI: 10.1115/1.1645877.

31. Investigation of the correlation between acoustic anisotropy, damage and measures of the stress-strain state / A. K. Belyaev, V. A. Polyanskiy, A. S. Semenov, D. A. Tretyakov, Y. A. Yakovlev // Procedia Structural Integrity. - 2017. - Vol. 6. - P. 201-207. DOI: 10.1016/j.prostr.2017.11.031.

32. O'Neill B., Maev R. G. Acousto-elastic measurement of the fatigue damage in Waspaloy // Research in Nondestructive Evaluation. - 2006. - Vol. 17, no. 3. - P. 121-135. DOI: $10.1080 / 09349840600787931$.

33. The formation of edge cracks during rolling of metal sheet / H. Riedel, F. Andrieux, T. Walde, K. F. Karhausen // Steel Research International. - 2007. - Vol. 78, no. 10-11. P. 818-824. - DOI: 10.1002/srin.200706291.

34. Ivanova Y., Partalin T., Pashkuleva D. Acoustic investigations of the steel samples deformation during the tensile // Russian Journal of Nondestructive Testing. - 2017. - Vol. 53, no. 1. P. 39-50. - DOI: 10.1134/S1061830917010077.

35. Kurashkin K. V., Gonchar A. V. Variation of acoustic characteristics of an aluminum alloy during plastic deformation at room and subzero temperatures // AIP Conference Proceedings. AIP Publishing, 2018. - Vol. 2053, no. 1. - P. 030030. - DOI: 10.1063/1.5084391.

36. Propagation of acoustic waves during the control of hydrogen-induced destruction of metals by the acoustoelastic effect / A. A. Alhimenko, A. K. Belyaev, A. I. Grishchenko, A. S. Semenov, D. A. Tretyakov, V. A. Polyanskiy, Y. A. Yakovlev // 2018 Days on Diffraction (DD). - IEEE, 2018. - P. 11-16. - DOI: 10.1109/DD.2018.8553151.

37. Acoustic anisotropy and dissolved hydrogen as an indicator of waves of plastic deformation / A. K. Belyaev, A. I. Grishchenko, V. A. Polyanskiy, A. S. Semenov, D. A. Tretyakov, L. V. Shtukin, D. G. Arseniev, Y. A. Yakovlev // 2017 Days on Diffraction (DD). - IEEE, 2017. P. 39-44. - DOI: 10.1109/DD.2017.8167992.

38. Effect of Surface Layer Damage on Acoustic Anisotropy / A. S. Semenov, V. A. Polyanskii, L. V. Shtukin, D. A. Tretyakov // Journal of Applied Mechanics and Technical Physics. - 2018. Vol. 59, no. 6. - P. 1136-1144. - DOI: 10.1134/S0021894418060202.

39. A study of hydrogen cracking in metals by the acoustoelasticity method / E. L. Alekseeva, A. K. Belyaev, L. A. Pasmanik, A. M. Polyanskiy, V. A. Polyanskiy, D. A. Tretiakov, Y. A. Yakovlev // AIP Conference Proceedings. - AIP Publishing, 2017. - Vol. 1915, no. 1. P. 030001. - DOI: 10.1063/1.5017321.

40. Boundary Layer of Hydrogen Concentration under Plastic Deformation / A. K. Belyaev, D. E. Mansyrev, V. A. Polyanskiy, A. M. Polyanskiy, D. A. Tretyakov, Yu. A. Yakovlev // Diagnostics, Resource and Mechanics of Materials and Structures. - 2017. - No. 4. - P. 32-43. - 
DOI: 10.17804/2410-9908.2017.4.032-043. - URL: http://dream-journal.org/issues/2017-4/20174_167.html

41. Influence of the skin effect of plastic deformation on hydrogen accumulation in metals / V. A. Polyanskiy, A. K. Belyaev, Y. A. Yakovlev, A. M. Polyanskiy, D. A. Tretyakov // AIP Conference Proceedings. - AIP Publishing, 2018. - Vol. 2053, no. 1. - P. 020011. DOI: $10.1063 / 1.5084357$.

42. Hydrogen diagnostics of metals and alloys / A. M. Polyanskiy, V. A. Polyanskiy, K. P. Frolova, Yu. A. Yakovlev // Diagnostics, Resource and Mechanics of Materials and Structures. - 2018. - No. 6. - P. 37-50. - DOI: 10.17804/2410-9908.2018.6.037-050. URL: http://dream-journal.org/DREAM_Issue_6_2018_Polyanskiy_A.M._et_al._037_050.pdf 43. ГОСТ Р. 52890-2007. Контроль неразрушающий. Акустический метод контроля напряжений в материале трубопроводов. Общие требования // М. : Стандартинформ, 2009. 